research and effective communication; but that negative critical thinking dispositions, like being conceptually disorganized or intellectually indifferent, or closedminded, or biased, can be harmful not only to one's own development and maturation as a practitioner, but can lead to poorer health care options for one's patients.

In sum, this reviewer says thumbs-up to this book. It is a first attempt, and a fine one. Sure one can find flaws and infelicities, and one might prefer that more was said about this and less about that. But in the end, this is a book that needed to be written. We need more books of this kind that attempt to show how to be more effective critical thinkers in a given area of professional practice. And we need books that help the faculty of professional schools more effectively teach for thinking. If someone can do a better job with evidence-based medical practice, let them step up and do it.

\author{
Peter Facione \\ University Provost and Professor of Philosophy \\ Office of the Provost \\ Loyola University Chicago \\ 820 North Michigan Avenue \\ Chicago, Illinois 60611 \\ Email:pfacion@luc.edu
}

\title{
Abduction, Reason, and Science: Processes of Discovery and Explanation
}

\section{Lorenzo Magnani}

New York, Boston, Dordrecht, London, Moscow: Kluwer Academic/ Plenum Publishers, 2001. pp.xvii, 1-205. ISBN 0-306-46514-0. \$93.50 USD.

\section{Reviewed by Marcello Guarini}

In the late 1980s, Paul Churchland!, Ronald Giere ${ }^{2}$, and Paul Thagard ${ }^{3}$ started applying insights gleaned from artificial intelligence, cognitive science, and psychology to problems in the philosophy of science. Those early works were characterized by extended, introductory discussions of the science being applied to philosophical problems. Things have changed since those pioneering efforts, and Lorenzo Magnani's Abduction, Reason, and Science is an example of the change. Gone are the introductory discussions of the science. Dozens of authors from artificial intelligence, cognitive science, philosophy, and psychology are cited, and the reader is expected to have at least some familiarity with this wide range of work. As a field develops, it is not uncommon for authors to expect more of their readers, so the 
style in which the book is written is not surprising. However, there are places where there is insufficient commentary on or development of the cited literature, making it difficult to track the argument. The preceding notwithstanding, the book contains original and important insights regarding abduction and its role in reasoning.

Magnani's monograph is primarily about abductive reasoning in science, but the views developed on abduction clearly have applications outside of science. He is concerned to establish that the abductive act of generating hypotheses can be understood as rational, and that the conceptual tools of the cognitive sciences can be useful in helping us to grasp how that is so. This view is contrasted with the positivist view that the rationality of science is to be found in the context of justification, not in the context of discovery (which is understood to include hypothesis generation) (p. xi). In Chapter One, we are treated to a tour-de-force discussion of Plato, Kant, and Polanyi. They are seen as historical examples of individuals who have treated hypothesis generation as "paradoxical, ... illusory or obscure, implicit, [or] not analyzable" (p. 1). The Meno dilemma-either you know what you are looking for, in which case there is no problem, or you do not know what you are looking for, in which case you will not find anything-motivates much of the chapter. Magnani appears to think that a generate and test model will allow us (a) to see that there is something we do not know and (b) to understand when we have learned what we did not initially know. Magnani uses the example of attempting to refute Golbach's conjecture to claim that we can define a problem when we are unsure what the solution is, and we can define procedures for testing whether proposed hypotheses are solutions (pp. 7-11). At this point, nothing has been said about hypothesis generation. Much of the rest of the book is an attempt to show that hypothesis generation can be modelled-that it can be laid open to our gaze (pace Kant) or rendered explicit (pace Polanyi).

In chapter two, Magnani distinguishes between two senses of the term 'abduction.' The first is concerned with the generation of plausible hypotheses for explaining some phenomena; the second amounts to a kind of inference to the best explanation that includes the process of generating plausible hypotheses but adds to it the process of evaluating and selecting the best of those hypotheses (pp. 19, 25). While the book is concerned with both senses of the term, the focus is on the generation of plausible hypotheses- the narrow sense of the term. Using th is narrow sense of the term, Magnani distinguishes between 'theoretical' and 'manipulative' abduction. Theoretical abduction "is the process of inferring certain facts and/or laws and hypotheses that render some sentences plausible, that explain or discover some (eventually new) phenomenon or observation; it is the process of reasoning in which explanatory hypotheses are formed and evaluated" (pp. 17-18). Chapter two is concerned with theoretical abduction, and chapter three with manipulative abduction (defined below). Two approaches to theoretical abduction are considered - sentential and model-based. Sentential models are, very roughly, applied formal logic (whether monotonic or nonmonotonic) approaches to understanding abduction. Model-based approaches to reasoning refer to "the construction and manipulation of various kinds of representations, not necessarily sentential and/or formal" (p. 45). Magnani examines different research programs in sentential 
abduction, observes that they tend to be concerned more with inference to the best explanation and less with hypothesis generation, and concludes that they are inadequate in accounting for the creative, hypothesis-generating moment in scientific reasoning. Different model-based approaches are examined and are found to have greater potential for explaining how scientific reasoning takes place, including hypothesis generation.

In chapter three, manipulative abduction is taken up. "Manipulative abduction refers to an extra-theoretical behavior that aims at creating communicable accounts of new experiences to integrate them into previously existing systems of experimental and linguistic (theoretical) practices" (p. 53). It involves the use of objects (hands, images, scale models, telescopes, etc...) to aid reasoning. The manipulation in question may involve physical movement in the external world, or purely internal or visualized manipulation of objects (before the mind's eye, as it were). Examples from the history of science are discussed to show how important such reasoning has been. Examples from "everyday life" are also discussed (p. 60). While visual abduction is introduced in chapter two as a form of manipulative abduction (p. 43), it only receives brief mention in the third chapter. A detailed treatment comes in chapter five.

Chapter four contains a detailed account of a medical diagnostic reasoning program, called NEOANEMIA, that implements Magnani's model-based select and test approach to abductive reasoning. The first step of this select and test approach requires that a set of plausible hypotheses be selected, where each hypothesis may explain the observed data. The hypotheses are ranked, and consequences are deduced from each of them. New information is requested and compared to the deduced consequences, leading to the corroboration or elimination of hypotheses, and to the re-ranking of existing hypotheses and the selection of new ones (if needed). The process of deducing consequences and checking the modified hypothesis set may be repeated as needed.

This chapter also engages the nature of the relationship between descriptive and prescriptive inquiry. Some might hold that psychology is descriptive and that bgic and epistem obgy are norm ative, so they should notbe m ixed-th is is not Magnani's view (pp. 52,77). Paul Thagard is cited endorsingly as pointing out that some models of reasoning may be so complex that computationally implementing them is a helpful way of figuring out what their implications are (p. 78). However, it is not always clear how Magnani intends to develop this point. Paul Thagard has defended the use of a biscriptive methodology, where the computational theories put forward are attempts to describe how people reason when they reason at their best-combining both descriptive and prescriptive/normative components to the study of reason. ${ }^{5}$ In other words, biscriptive computational models correctly describe how people reason when they reason in a normatively acceptable manner. While such models need not reduce the normative to the descriptive, they cannot make use of processes that exceed human abilities on pain of being descriptively inadequate. Magnani does not come out and endorse a biscriptive approach; in fact, there is even a passage where he suggests that describing human performance need not be a goal of useful computational models (p. 84). He does seem to think 
that computational models can help us to understand why we deviate from epistemological principles (p. 78), but does that deviation mean the principles should change, that we should rethink the cogency of the principles? Or does it mean that we should continue to use existing epistemic standards and make use of computational models (a) as a guide in diagnosing why our performance falls short of those standards and (b) as a tool for figuring out how to improve our performance? The end of the chapter attempts to draw out some of the educational benefits of computational models, suggesting that something like (a) and (b) might be what he has in mind. If that is right, perhaps computational models should be taken as biscriptive, as an attempt to describe how we actually reason when we reason at our best. If they are not biscriptive, why should we use them as a guide for correcting our reasoning? After all, they may embody capacities (pertaining to speed of processing and memory) that exceed human abilities, in which case it is not clear how they could be normatively useful. (You would not tell someone that she ought to learn how to play a piano chord with 16 notes; it is not within a human's powers to do so. Similarly, it is not clear why anyone would say to an epistemic agent that she ought to learn how to process or use memory in a way that is beyond the ability of a human being.) To his credit, Magnani recognizes the importance of discussing the relationship between descriptive and normative issues; however, it would have been helpful to see more said on the matter.

Chapter five has two themes-visual abduction and temporal abduction. Magnani takes an image to be a kind of "internal representation used by humans to retrieve information from memory" (p. 97). Literature is surveyed pertaining to the use of images in reasoning both inside and outside of science, and a general architecture is proposed for how to account for such reasoning. Magnani endorses a kind of hybrid imagery/linguistic representation architecture. He does not believe that the images in this architecture express information that the linguistic representations cannot; rather, he thinks that images encode information in such a way that using them greatly reduces the computational complexity of arriving at a solution (p. 105). Images are useful both for the generation of plausible hypotheses (visual abduction) and for the evaluation of those hypotheses. Reasoning about the history of an object and how it changes over time will require that time be explicitly represented-both in image-based reasoning and in other forms of reasoning. Since we frequently make temporal assumptions about objects when generating plausible explanations, and since anomalous results sometimes force us to rethink those temporal assumptions, Magnani reasons that time needs to be represented in models of hypothesis generation and evaluation. While the terms "visual abduction" and "temporal abduction" are treated in different sections of this chapter, they are not unrelated. Adding a representation of time to a visual reasoning system (adding temporal constraints to imagistic constraints in hypothesis generation and evaluation) may greatly improve the efficiency of that system and the range of problems it can handle.

Chapter six motivates the need for dealing with various types of inconsistencies. The different ways that inconsistencies may arise are discussed, and a modelbased approach to dealing with inconsistencies is argued for over purely logical 
Book Reviews/Comptes Rendus 305

(sentential) approaches (p. 130). The issue of how to deal with inconsistencies is treated further in chapter seven, entitled "Hypothesis Withdrawal in Science." Magnani claims that some hypotheses may be difficult or impossible to falsify, but there may still be a place for them in scientific theorizing. The key to seeing this is the principle of negation as failure. Roughly, if all attempts to prove $P$ fail, we are allowed to infer not-P (p. 147). For example, if a theory we believe is committed to $P$, and all attempts to prove $P$ fail, then we infer not-P. Failure to prove can generate an inconsistency, which we then need to deal with. Negation as failure is applied to Freudian psychoanalysis. Say an analyst comes up with a construction-a kind of retrodiction-of the analysand's early life experiences. The analyst may then seek further information from the patient in an attempt to prove that the construction is legitimate. If all further information received from the analysand fails to support the construction, then the construction must be given up. $\mathrm{P}$ was abduced as an explanation of some phenomena; all attempts to further support $P$ failed, so not-P is inferred and other hypotheses must be abduced. Magnani attempts to show that Freud himself endorsed a procedure more or less like the one just described (pp. 149-155). The principle of negation as failure is applied to Poincaré as well. Of course, the principle (which can generate inconsistencies in a belief set) should be read against the background of Chapter Six, where Magnani argues that there may be times when inconsistencies need to be "maintained" or tolerated in light of considerations such as overall coherence or the absence of a successor theory (pp. 136-139).

Many will find this book a thought-provoking read. The treatment of visual reasoning as a species of manipulative abduction, and the presentation of the model-based approach as an alternative to purely formal-logic based approaches should prove especially interesting to our readership.

\section{Notes}

' Paul Churchland, A Neurocomputational Perspective: The Nature of Mind and the Structure of Science (MIT Press, 1989).

${ }^{2}$ Ronald N. Giere, Explaining Science: A Cognitive Approach (University of Chicago Press, 1988).

${ }^{3}$ Paul Thagard, Computational Philosophy of Science (MIT Press, 1988).

${ }^{4}$ The refutation in question would involve finding an even number that is not the sum of two prime numbers.

${ }^{5}$ Paul Thagard, Conceptual Revolutions (Princeton University Press, 1992): 97.

Marcello Guarini

Department of Philosophy

University of Windsor

Windsor, Ontario

Canada. N9B $3 P 4$ 